\title{
Wild-type p53 suppresses the epithelial-mesenchymal transition and stemness in PC-3 prostate cancer cells by modulating miR-145
}

\author{
DONG REN $^{1}$, MIN WANG ${ }^{1}$, WEI GUO ${ }^{1}$, XIAOHUI ZHAO ${ }^{2}$, XIANG'AN TU $^{3}$, \\ SHUAI HUANG ${ }^{1}$, XUENONG ZOU $^{1}$ and XINSHENG PENG ${ }^{1}$ \\ ${ }^{1}$ Department of Orthopaedic Surgery, The First Affiliated Hospital of Sun Yat-sen University, Guangzhou 510080; \\ ${ }^{2}$ Department of Experimental Research, State Key Laboratory of Oncology in Southern China, \\ Sun Yat-sen University Cancer Center, Guangzhou 510060; ${ }^{3}$ Department of Urology, \\ The First Affiliated Hospital of Sun Yat-sen University, Guangzhou 510080, P.R. China
}

Received December 1, 2012; Accepted January 28, 2013

DOI: $10.3892 /$ ijo.2013.1825

\begin{abstract}
The principal problem arising from prostate cancer $(\mathrm{PCa})$ is its propensity to metastasize to bone and the mechanism(s) need to be further elucidated. The tumor suppressor p53 plays an important role in regulating the epithelial-mesenchymal transition (EMT) and cancer cell stemness, which have been proposed to play critical roles in cancer metastasis. MiR-145, a direct target of p53, represses bone metastasis of $\mathrm{PCa}$ and is involved in regulating EMT and cancer cell stemness. However, it is unknown whether wild-type p53 (WT-p53) plays a role in regulating invasion, EMT and cancer cell stemness of PCa cells and whether miR-145 mediates the function of WT-p53. In the present study, we found that ectopic expression of WT-p53 inhibited the migration and invasion, and enhanced the adhesion of p53-null PC-3 cells derived from PCa bone metastasis. Furthermore, WT-p53 suppressed the expression of the mesenchymal markers fibronectin, vimentin, $\mathrm{N}$-cadherin, ZEB2 and upregulated the expression of the epithelial marker E-cadherin in PC-3 cells. Moreover, WT-p53 also suppressed colony formation, tumor sphere formation and expression of CSC markers and stemness factors including CD44, Oct4, c-Myc and Klf4 in PC-3 cells. Importantly, WT-p53 upregulated the expression of miR-145, and the inhibitory
\end{abstract}

Correspondence to: Professor Xinsheng Peng, Department of Orthopaedic Surgery, The First Affiliated Hospital of Sun Yat-sen University, 58 Zhongshan 2nd Road, Guangzhou, Guangdong 510080, P.R. China

E-mail: pengxs66@yahoo.com

Abbreviations: PCa, prostate cancer; miRNAs, microRNAs; EMT, epithelial-mesenchymal transition; CSCs, cancer stem cells; WT-p53, wild-type $\mathrm{p} 53$

Key words: prostate cancer, wild-type p53, microRNAs, epithelialmesenchymal transition, cancer cell stemness effects of WT-p53 on migration, invasion, EMT and stemness of PC-3 cells were reversed by anti-miR-145. Together, our findings demonstrate that WT-p53 suppresses migration, invasion, EMT and stemness in PC-3 cells at least partially through modulating miR-145. These results suggest that loss of WT-p53 may promote the bone metastasis of PCa at least partially through repressing miR-145 to elevate EMT and stemness of cancer cells.

\section{Introduction}

The principal issue derived from prostate cancer (PCa) is its inclination to metastasize to bone, which occurred in as many as $90 \%$ of patients with advanced PCa (1). However, the exact mechanisms of bone metastasis of PCa need further to be elucidated.

As a transcription factor, the tumor suppressor p53 mediates changes in gene expression that promote apoptosis, senescence or a reversible and protective cell cycle arrest $(2,3)$. In about half of all human cancers, p53 is either lost or mutated. Loss of wild-type p53 (WT-p53) function is well known to influence cell cycle checkpoint controls and apoptosis (4) and gain of function of mutant p53 is involved in development and progression of many cancers (4-6). Importantly, emerging evidence has shown that WT-p53 also plays a role in regulating key stages of metastatic progression $(4,6)$, but how it functions as metastasis/invasion suppressor is just beginning to be understood (7). In PCa, it also remains elusive whether and how WT-p53 regulates bone metastasis although mutant p53 may promote bone metastasis (5).

Recent studies have found that two important mechanisms by which p53 regulates metastasis, repression of migration and invasion of cancer cells through modulating epithelial-mesenchymal transition (EMT) and suppression of cancer stem cell (CSC) properties (4,8-11). EMT is a key step of the progression of tumor cell metastasis (12). It also has been identified as an important step in bone metastasis of PCa (13). E-cadherin plays a critical role in EMT which is regulated by transcription factors including Snail, Slug, Twist and Zeb1/2 (14). By targeting these transcription factors, p53 
regulates EMT (6). Furthermore, the CSCs are the cells within tumors that possess the ability of self-renewal, immortalized proliferation and differentiate into the heterogeneous lineages of cancer cells which consist of the whole tumor $(15,16)$. These capabilities of cancer stem cells have formed the basic definition of 'stemness' (17). Accumulating evidence suggests that cancer cell stemness is associated with the metastasis of tumors $(16,17)$. Recent studies indicated that p53 has crucial influence on cancer cell stemness by regulating key stemness genes $(8,11)$.

Emerging evidence has demonstrated that miRNAs are components of the cellular signaling circuitry that regulates the EMT program (18), such as miR-200 family $(19,20)$, miR-34 family $(21,22)$ and miR-205 (19). These miRNAs directly target transcription factors Snail, Slug and Zeb1/2, and regulate the EMT of cancer cells. Furthermore, miRNAs also played a pivotal role in regulating the properties of CSCs by negatively regulating the expression of certain key genes, such as CD44, Oct4, Sox2, c-Myc and Klf4 (23). Moreover, some miRNAs are transcriptionally regulated by p53 (24). Importantly, several miRNAs, such as miR-200c and miR-34 family mediate p53 regulation of EMT (8-11) and stem cell properties in cancers (21).

Our previous studies have demonstrated that miR-145 is associated with bone metastasis of PCa by suppressing EMT and stemness of cancer cells $(25,26)$. Also microRNA-145 is directly regulated by WT-p53 (27-30), and the loss of WT-p53 function occurs in many $\mathrm{PCa}$, therefore, we reasoned that WT-p53 may play a role in regulating EMT and cancer cell stemness of PCa cells and miR-145 may mediate the function of WT-p53.

To test the hypothesis, we upregulated expression of WT-p53 in p53-null PC-3 cells derived from PCa bone metastasis and found that ectopic expression of WT-p53 inhibited migration, invasion, EMT and stemness of PC-3 cells, and the inhibitory effects of WT-p53 on EMT and cancer cell stemness of PC-3 cells were reversed by anti-miR-145. Our findings demonstrate that WT-p53 represses EMT and stemness of PC-3 cells at least partially by mediating the miR-145.

\section{Materials and methods}

Cell culture. The bone metastatic PCa cell line PC-3 was purchased from American Type Culture Collection (ATCC, Manassas, VA, USA) and grown in Ham's F-12 culture medium (HyClone, Logan, UT, USA) supplemented with $10 \%$ fetal bovine serum (HyClone). Cells were grown at a humidified atmosphere of $5 \% \mathrm{CO}_{2}$ at $37^{\circ} \mathrm{C}$.

Plasmids and transient transfection. Plasmids expressing WT-P53 and miR-145-antagomiR were purchased from RiboBio Co. Ltd (Ribo, China). The cloning sequence of WT-P53 was from 203 to 1,384 in the CDS region and miR-145 in pMSCV was constructed as described previously (25). Before transfection, $2 \times 10^{5}$ cells were seeded into each well of 6 -well plates. After $24 \mathrm{~h}$ incubation in growth medium, the cells were transiently transfected with using Lipofectamine 2000 (Invitrogen, Carlsbad, CA, USA) according to the manufacturer's protocol. Transfection medium is the Opti-Mem medium which is used for transient transfection. The trans- fection medium was removed after 4-6 h, and the cells were incubated for an additional $48 \mathrm{~h}$ in $10 \%$ fetal bovine serum medium ( $2 \mathrm{ml}$ per well).

Quantitative reverse transcription-PCR. The procedure was carried out according to the manuscript of All-in-One ${ }^{\mathrm{TM}}$ miRNA qRT-PCR detection kit (GeneCopoeia, Rockville, MD, USA). Total RNAs were extracted from cells by using RNeasy kit (Qiagen). Total RNA was reverse transcribed by adding poly-A sequence, and real-time PCR analysis was performed with specific primer to WT-p53 and hsa-miR-145 (GeneCopoeia). Each sample was analyzed in triplicate. No template, and no reverse transcription were included as negative controls. U6 snRNA was used as normalization control. Relative expression values from three independent experiments were calculated following the $2^{-\Delta \Delta C t}$ method of Schmittgen and Livak (31).

Western blot analysis. For the analysis of expression of related proteins, western blot assay was performed. The cells were seeded in 6-well plates. After 24-48 h, cells were washed with pre-chilled PBS and at confluence of 60-70\% harvested in sample buffer [62.5 mmol/1 Tris- $\mathrm{HCl}(\mathrm{pH} 6.8)$, $2 \%$ SDS, $10 \%$ glycerol and 5\% $\beta$-mercaptoethanol]. Equal amounts of protein from the supernatant were loaded per lane and resolved by SDS-polyacrylamide electrophoresis. Then, protein was transferred onto PVDF membrane (Millipore), blocked by $5 \%$ non-fat milk for $1 \mathrm{~h}$ at room temperature, and probed with primary antibodies $(1: 1,000)$ overnight at $4^{\circ} \mathrm{C}$, including rabbit anti-P53, Oct4, c-Myc, Klf4, CD44 and mouse anti-E-cadherin, vimentin (Cell Signaling Technology); rabbit anti-N-cadherin (Millipore); mouse antifibronectin (BD Biosciences) and mouse anti-ZEB2 (Sigma, St. Louis, MO, USA). Membranes were washed three times (10 min each) in TBS-T buffer and incubated for $40 \mathrm{~min}$ at room temperature with horseradish peroxidase-conjugated anti-mouse or anti-rabbit secondary antibodies. Membranes were washed thrice (10 min each) in TBS-T and developed using the ECL system. Protein loading was normalized by reprobing the blots with rabbit anti- $\beta$-actin (Cell Signaling Technology).

Wound healing assay. PC-3 cells were trypsinized and seeded equivalently into 6-well tissue culture plates $24 \mathrm{~h}$ before scratching, and grew to reach almost total confluence in $24 \mathrm{~h}$. Non-serum starvation lasted for $24 \mathrm{~h}$. After cell monolayers formed, a wound was scratched onto the monolayer with a sterile $100 \mu \mathrm{l}$ tip (Axygen, Union City, CA, USA). After scratching, the cells were washed with PBS twice and cultured in $10 \%$ fetal bovine serum media. Images of PC-3 cells migrating into the wound were captured at time points of 0,6 and $12 \mathrm{~h}$ by an inverted microscope $(\mathrm{x} 40)$.

Invasion assay. The invasion assay was performed by using Transwell chamber consisting of 8-mm membrane filter inserts (Corning) coated with Matrigel (BD Biosciences). Briefly, the cells were trypsinized and suspended in serum-free medium. Then $1.5 \times 10^{5}$ cells were added to the upper chamber, and lower chamber was filled with the medium with $10 \%$ FBS. After incubated for 24-48 h, cells passed through the coated 
membrane to the lower surface, in which cells were fixed with $4 \%$ paraformaldehyde and stained with hematoxylin. The cell count was done under a microscope (x100).

Adhesion assay. Briefly, 96-well plates were coated with $50 \mu 1$ fibronectin $(50 \mu \mathrm{g} / \mathrm{ml})$ at cell incubator for $1 \mathrm{~h}$. After washed with warm PBS, the plates were blocked with $1 \%$ BSA at $37^{\circ} \mathrm{C}$ for $1 \mathrm{~h}$ and washed twice. After trypsinization, suspended cells were seeded to each well with serum-free media at a density of $1.5 \times 10^{4}$ cells per well. The cells were incubated for $30 \mathrm{~min}$, non-adherent cells were removed and plates were gently washed twice with PBS. Adherent cells were fixed in 4\% paraformaldehyde for $20 \mathrm{~min}$ at room temperature, then stained with hematoxylin and counted under an inverted microscope (x100).

Colony formation assay. The cells were trypsinized as single cells and suspended in serum-free medium. Cells (300 cells/ml) were seeded into each well of 6-well plates for 1-2 weeks, and colonies were dyed with crystal violet. Plating efficiency $=$ number of colonies ( $\geq 50$ cells per colony) per input cells $\mathrm{x} 100 \%$. Colony morphology was determined, by scoring under a light microscope.

Self-renewing spheroid formation assay. Cells (500 cells/ well and 50-100 cells/well) were plated respectively onto 6-well and 24-well Ultra Low Cluster plate (Corning) and were cultured in suspension in serum-free DMEM-F12 (BioWhittaker), supplemented with B27 (1:50, Invitrogen), $20 \mathrm{ng} / \mathrm{ml}$ EGF (BD Biosciences), $0.4 \%$ bovine serum albumin (Sigma), and $4 \mathrm{mg} / \mathrm{ml}$ insulin (Sigma) for 10-14 days. After 10-14 days, the number of PC-3 cell spheres (tight, spherical, non-adherent masses $>50 \mu \mathrm{m}$ in diameter) were counted, and image of the spheres were captured under an inverse microscope. Sphere formation efficiency $=$ colonies/input cells $\mathrm{x}$ $100 \%$.

Statistical analyses. Statistical significance of the studies was analyzed by Student's t-test or one-way ANOVA. Statistical significance was accepted at $\mathrm{p}<0.05$.

\section{Results}

WT-p53 repressed invasiveness of PC-3 cells and anti-miR-145 rescued the effect. To investigate the role of WT-p53 in the development and progression of PCa metastasis, we upregulated WT-p53 by transfecting the plasmid of WT-p53 expression in PC-3 cells, in which p53 is null. The expression of WT-p53 was confirmed by real-time PCR in mRNA level (Fig. 1A) and western blot analysis in protein level (Fig. 1B). We investigated whether WT-p53 was able to regulate invasion, migration and adhesion in PC-3 cells. By using Transwell Matrigel invasion assay to assess the invasive ability of cells, we found that WT-p53 repressed invasive ability to $45.7 \%$ of PC-3/vector (Fig. 1C). In adhesion assay, WT-p53 increased adhesive ability 2.87-fold that of PC-3/vector (Fig. 1D). As shown in Fig. 1E, in cell migration observed by wound healing assay, WT-p53 decreased the healing speed of the cell wound compared to the PC-3/ vector.
Because miR-145 is transcriptionally regulated by WT-p53 (27-30), determined whether inhibiting miR-145 could rescue the effects of WT-p53. After upregulating WT-p53, we applied miR-145-antagomiR in PC-3 cells. The expression of miR-145 was confirmed by real-time PCR. We found that WT-p53 upregulated the expression of miR-145 31.0-fold that of the PC-3/ vector (Fig. 1F). The anti-miR-145 repressed WT-p53 expression in mRNA and protein levels. It also completely counteracted expression of WT-p53 in PC-3 cells as anti-miR-145 increased the cell migration speed and invasive ability that was reduced by WT-p53 expression, and also reduced miR-145 expression and the adhesive ability that was increased by WT-p53 (Fig. 1). These results indicated that WT-p53 inhibited invasiveness of PC-3 cells through modulation of miR-145.

WT-p53 represses EMT and anti-miR-145 rescues the effect in $P C-3$ cells. To investigate whether miR-145 regulated invasiveness by repressing EMT, we examined the influence of ectopic expression of WT-p53 on expressions of E-cadherin, $\mathrm{N}$-cadherin, fibronectin, vimentin and ZEB2 of PC-3 cells by western blot analysis. E-cadherin, which is one of key epithelial markers and supposed to be downregulated during EMT, was increased in PC-3 cells with WT-p53 expression. $\mathrm{N}$-cadherin, fibronectin and vimentin, which are kind of mesenchymal markers and should be upregulated during EMT, were repressed in PC-3 cells with WT-p53 expression. ZEB2, which is a transcription factor upregulating EMT, was repressed in PC-3 cells with WT-p53 expression (Fig. 2A and $\mathrm{B}$ ). Further, we detected the change of morphology of PC-3 cell with characteristics of EMT. The PC-3 cells with ectopic expression of WT-p53 converted the predominant mechenchymal phenotype to an evident epithelial phenotype i.e. from a stick-like or long spindle-shaped mesenchymal profile to a cobblestone-like or a short spindle-shaped epithelial morphology (Fig. 2C).

We determined whether inhibiting miR-145 would rescue the effects of expressing WT-p53 in PC-3 cell. As Fig. 2 shows, the anti-miR-145 completely counteracted the effects of WT-p53 expression in PC-3 cells as anti-miR-145 reduced the level of the epithelial cell marker E-cadherin that was increased by p53 expression, and restored expression levels of the mesenchymal cell marker $\mathrm{N}$-cadherin, fibronectin, vimentin and ZEB2 that was reduced by WT-p53 expression. The anti-miR-145 also was able to convert the predominant epithelial phenotype that was changed by WT-p53 expression to an evident mechenchymal phenotype. These observations indicated that WT-p53 repressed EMT through modulation of miR-145 in PC-3 cells.

WT-p53 inhibits colony formation of PC-3 cells and anti-miR-145 rescues the effect. We determined efficiency of WT-p53 inhibiting colony-forming of PC-3 cells in vitro. The colony-forming assay was performed. The number of colonies (\% plating efficiency) were $34.3 \%$ in PC-3/WT-p53 and $63.7 \%$ in PC-3/vector and significantly decreased in PC-3/WT-p53 compared with $\mathrm{PC}-3 /$ vector $(\mathrm{p}<0.01$, respectively) (Fig. 3 ). Colonies with different morphologies in vitro are classified as holoclones, meroclones and paraclones (32). Holoclones are generally more round and tightly packed; paraclones are irregular in composition and often contain more elongated or 
A

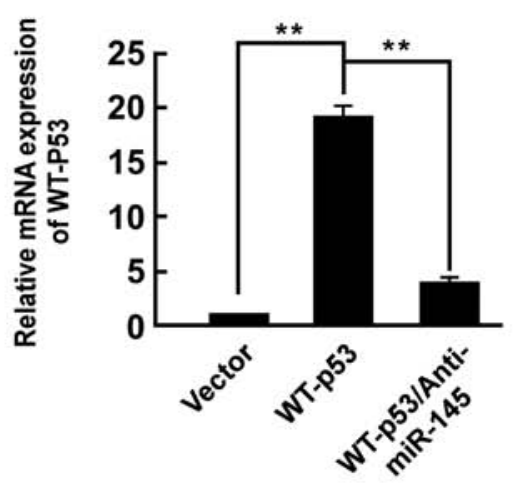

C

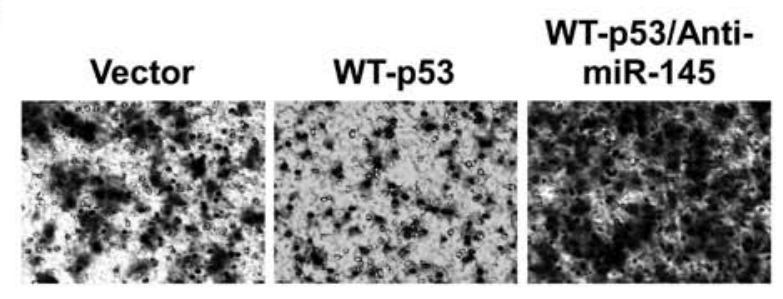

D

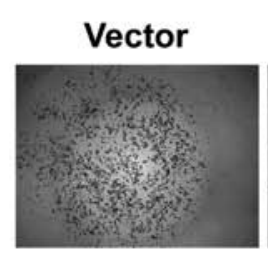

WT-p53

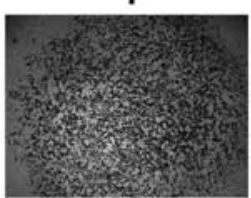

E

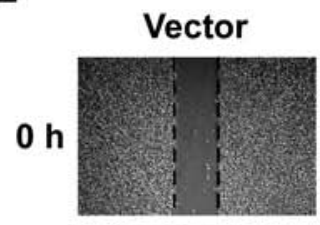

$6 \mathrm{~h}$

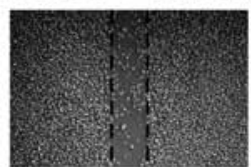

$12 \mathrm{~h}$

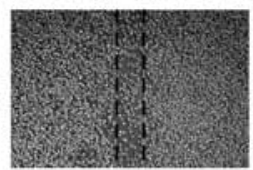

B miR-145

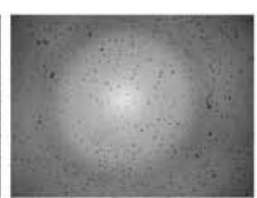
miR-145
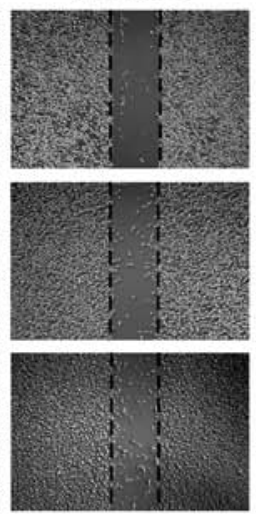
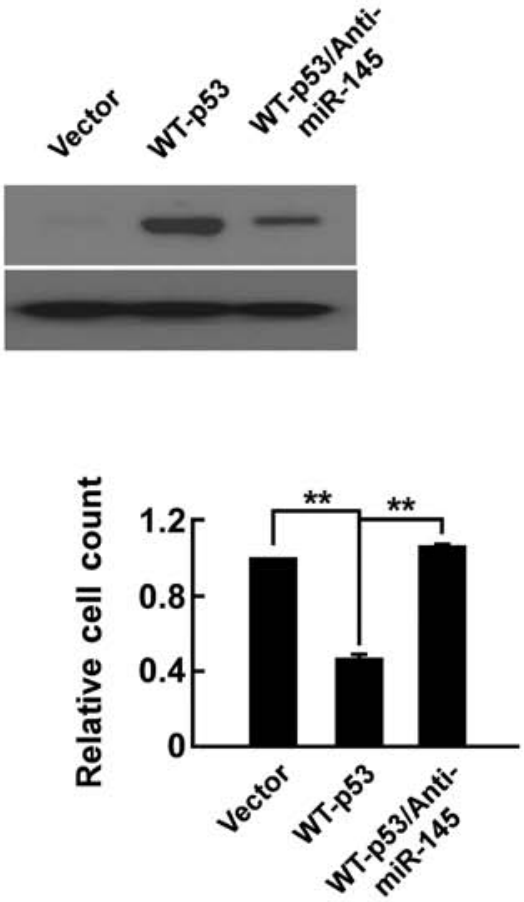

WT-p53/Anti-

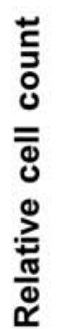

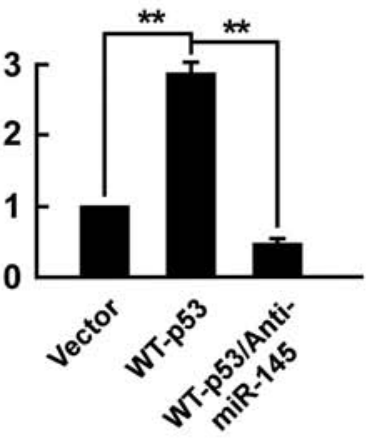

WT-p53/Anti- F
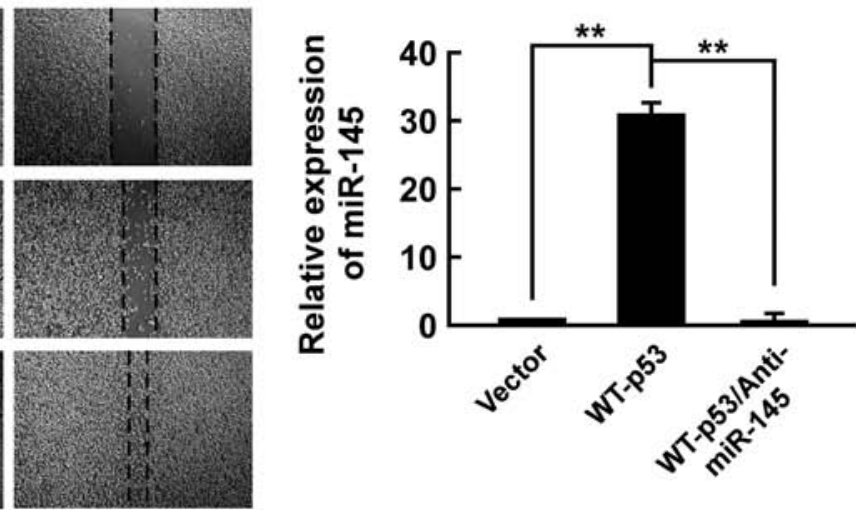

Figure 1. WT-p53 repressed invasiveness of PC-3 cells by modulating miR-145 in PC-3 cells. (A) The mRNA level of WT-p53 in PC-3/vector, PC-3/WT-p53 and PC-3/WT-p53/anti-miR-145. (B) WT-p53 protein level in PC-3/vector, PC-3/WT-p53 and PC-3/WT-p53/anti-miR-145. (C, D and E) WT-p53 efficiently suppressed migration and invasion; increased adhesion in PC-3 cells; and anti-miR-145 counteracted the effects of WT-p53 (" p $<0.01)$.

flattened cells; and meroclones are an intermediate phenotype. We did not find typical holoclones in PC-3 cells. The proportion of meroclones was $80.1 \%$ in $\mathrm{PC}-3 /$ vector and $58.9 \%$ in PC-3/WT-p53, and WT-p53 significantly decreased the proportion of meroclones of PC-3 cells $(\mathrm{p}<0.01$, respectively) (Fig. 3). Furthermore, we determined whether
WT-p53 regulates colony-forming by modulating miR-145 in PC-3 cells. As shown in Fig. 3, the anti-miR-145 completely counteracted the effect of expressing WT-p53 in PC-3 cells as anti-miR-145 restored colony-forming capability that was diminished by WT-p53 expression. The number of colonies was $71.4 \%$ and the proportion of meroclones was $81.4 \%$ 
A

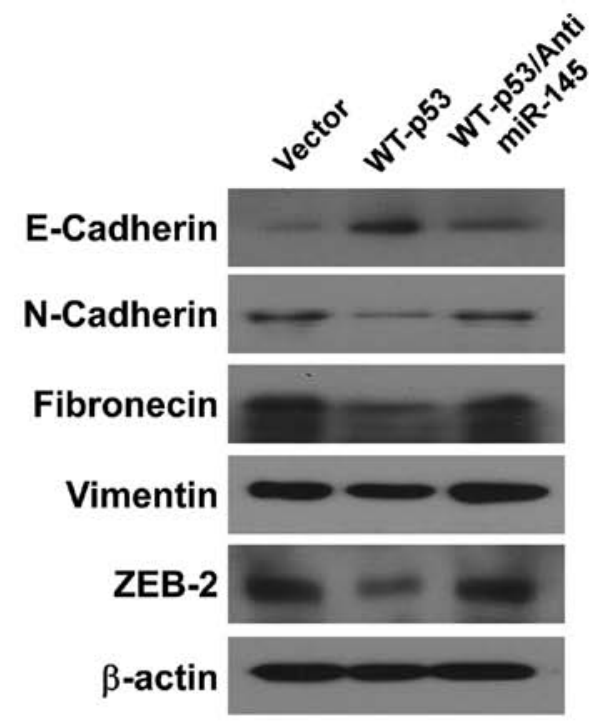

B
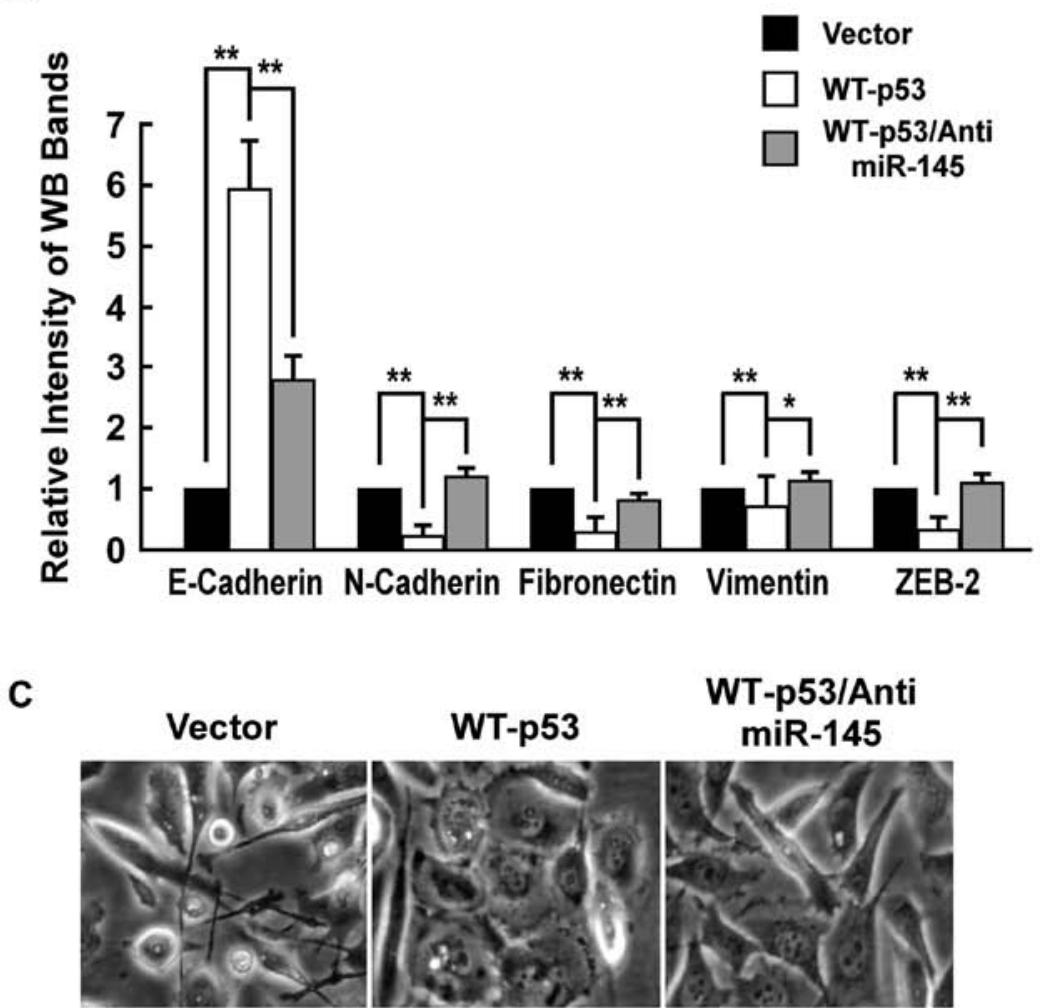

Figure 2. WT-p53 represses EMT by modulating miR-145 in PC-3 cells. (A and B) WT-p53 increased E-cadherin expression and suppressed N-cadherin, fibronectin, vimentin and ZEB2 expression and anti-miR-145 counteracted the effects of WT-p53 ("p<0.05; $\left.{ }^{*} \mathrm{p}<0.01\right)$. (C) WT-p53 converted a stick-like or long spindle-shaped mesenchymal profile to a cobblestone-like or a short spindle-shaped epithelial morphology, and anti-miR-145 counteracted the effects of WT-p53.

in PC-3/WT-p53/anti-miR-145. These data indicated that WT-p53 repressed cancer cell colony-forming by modulation of miR-145 in PC-3 cells.

WT-p53 inhibits tumor spheroid formation of PC-3 cells and anti-miR-145 rescues the effect. The ability to grow as non-adherent spheroids in the sphere medium has been widely used to assess the self-renewal capability of CSCs and is one of the properties of prostate CSCs (33). To confirm that WT-p53 can inhibit the self-renewal capability of PC-3 cells, prostasphere formation of PC-3 cells was studied. As shown in Fig. 4, after culturing for 12 days under non-adherent conditions, there were prostaspheres in all the cell types. The spheroid formation efficiency was $4.8 \%$ in PC-3/vector and $2.3 \%$ in PC-3/ WT-p53, confirming the presence of the self-renewal cell in PC-3/vector and PC-3/WT-p53, and WT-p53 suppressed significantly prostasphere formation $(\mathrm{p}<0.05)$. Furthermore, we determined whether WT-p53 regulates tumor spheroid formation by modulating miR-145 in PC-3 cells. As shown in Fig. 4, the anti-miR-145 completely counteracted the effect of WT-p53 expression in PC-3 cells as anti-miR-145 restored the capability of tumor spheroid formation that was diminished 
A

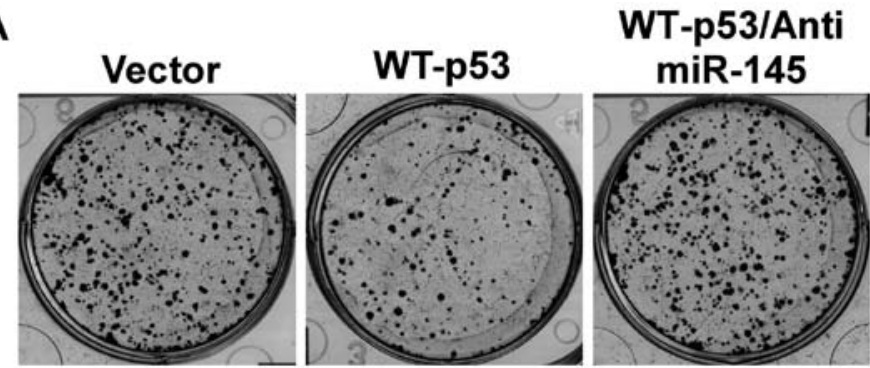

B

Meroclone

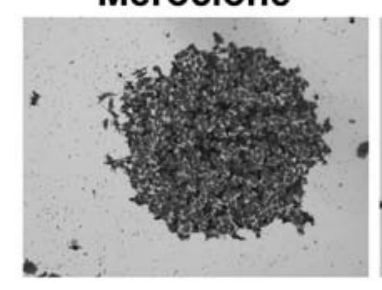

C

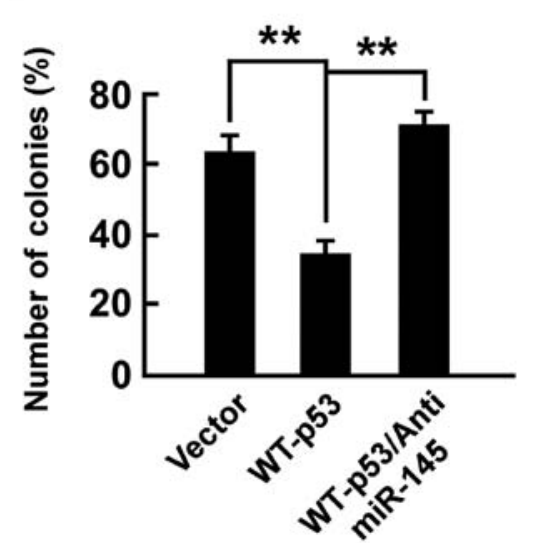

Paraclone

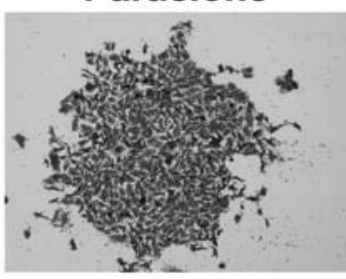

D

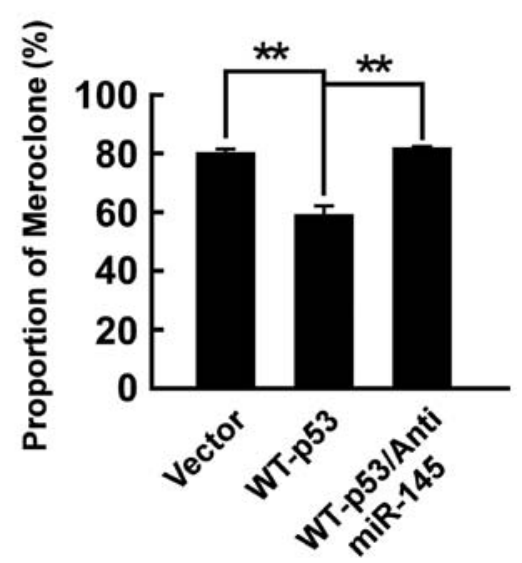

Figure 3. WT-p53 inhibits colony formation of PC-3 cells by modulating miR-145. (A) Colony-formation existed in PC-3/vector, PC-3/WT-p53 and PC-3/WT-p53/anti-miR-145. (B) A typical holoclone phenotype was not found, and only meroclones and paraclones were observed. (C and D) WT-p53 significantly repressed the number of colonies formation and the proportion of meroclones. The miR-145 reversed the effect of WT-p53 ( $\left.{ }^{* *} \mathrm{p}<0.01\right)$.

A

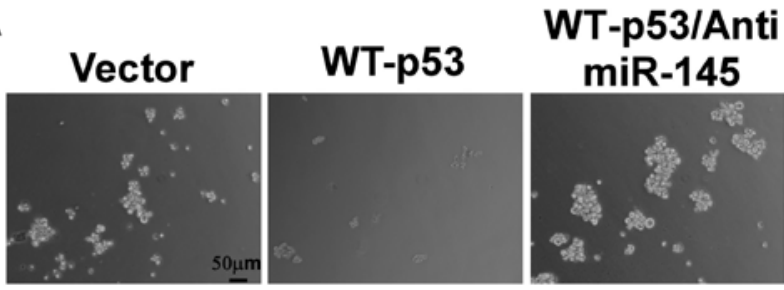

B

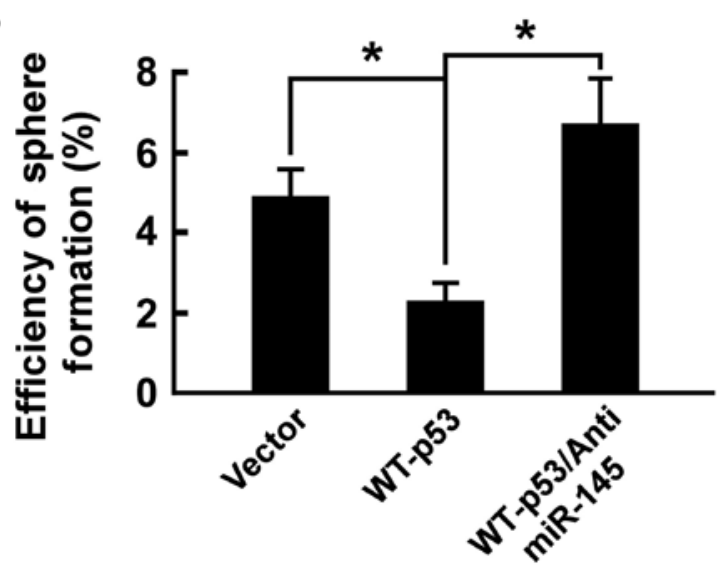

by WT-p53 expression. The spheroid formation efficiency was $6.7 \%$ in PC-3/WT-p53/anti-miR-145. These data indicated that WT-p53 repressed the self-renewal capability of cancer cells by modulating miR-145 in PC-3 cells.

WT-p53 inhibits CD44, c-Myc, Oct4 and Klf4 expression and anti-miR-145 restores their expression in PC-3 cells. To elucidate whether WT-p53 also have an influence on CSC marker and 'stemness' factor expression of PCa cells, we upregulated WT-p53 in PC-3 cells by transfecting the plasmid of WT-p53 expression and detected the expression of stem cell properties-associated factors and markers, including c-Myc, Oct4, Klf4 and CD44. As shown in Fig. 5, WT-p53 reduced the expression of CD44, which has been described as prostate CSC

Figure 4. WT-p53 suppresses the ability of tumor sphere formation in PC-3 cells by modulating miR-145. (A) Tumor sphere formation was observed by PC-3/vector, PC-3/WT-p53 and PC-3/WT-p53/anti-miR-145. (B) WT-p53 efficiently suppressed the ability of spheroid formation and miR-145 reversed the effect of WT-p53 ("p<0.05). 


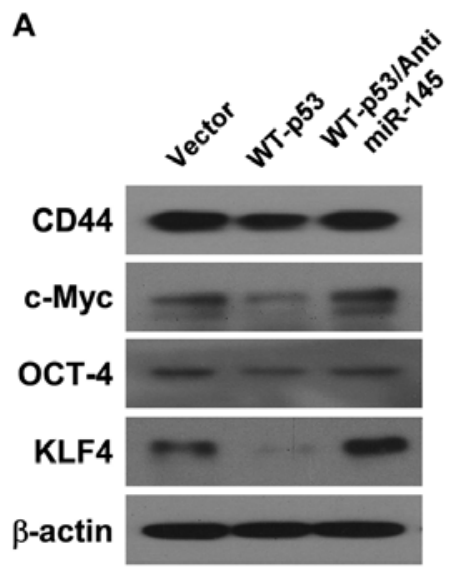

B

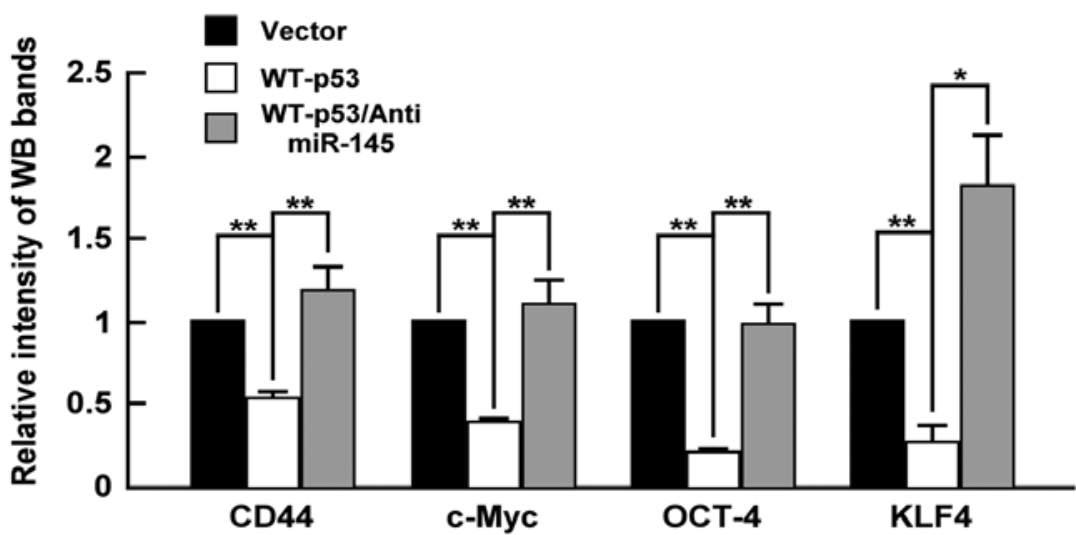

Figure 5. WT-p53 inhibits CD44, c-Myc, Oct4 and Klf4 expression in PC-3 cells by modulating miR-145. The overexpression of WT-p53 by transfecting the plasmids of WT-p53 significantly reduced the expression levels of CD44, C-Myc, Oct 4 and Klf4, and anti-miR-145 counteracted the effects of WT-p53 ("p<0.05; * p $<0.01$ ).

marker based on clinical investigations and in vitro studies of prostate cancer cell lines (21), and downregulated the expression of Oct4, c-Myc and Klf4, which are the key 'stemness' factors, and are required for maintaining self-renewal and pluripotency of stem cells (34). Furthermore, we determine whether WT-p53 regulates the stemness factors by modulating miR-145 in PC-3 cells, and found that anti-miR-145 counteracted the effects of WT-p53 expression as anti-miR-145 restored expression levels of CD44, Oct4, c-Myc and Klf4 that was reduced by WT-p53 expression (Fig. 5). These data indicated that WT-p53 repressed 'stemness' factors through modulation of miR-145 in PC-3 cells.

\section{Discussion}

We have previously identified that miR-145 may repress bone metastasis of PCa and is involved in regulating EMT and stemness of PCa cells $(25,26)$. In the present study, we found that the ectopic expression of WT-p53 inhibited migration, invasion, EMT, colony formation, tumor sphere formation and expression of CD44, Oct4, c-Myc and Klf4 in PC-3 PCa cells, and enhanced miR-145 expression in PC-3 cells. Importantly, the anti-miR-145 was able to reverse the above-mentioned inhibitory effects of WT-p53. Our findings demonstrate that WT-p53 repressed EMT and cancer cell stemness of PC-3 cells at least partially through regulation of miR-145.

Many studies have demonstrated that p53 plays a key role in the function of cell cycle, apoptosis, senescence, DNA-repair mechanisms and autophagy $(3,4)$. However, the emerging evidence demonstrates that p53 also plays a crucial role in regulating key stages of the metastatic progression $(4,6)$. In $\mathrm{PCa}$, although some studies have showed that mutant $\mathrm{p} 53$ gain of function promoted cancer development and metastasis $(5,35-$ 38), there are hardly any studies on the role of WT-p53 in PCa metastasis.We found that WT-p53 repressed invasion and migration, and suppressed EMT, which is a key step of the progression of tumor cell metastasis, and cancer stem cells properties in PC-3 cells, which might be the critical drivers of tumor progression and metastasis $(16,17)$. Zhou et al $(39,40)$ have found that the combined deficiency for $\mathrm{p} 53$ and $\mathrm{Rb}$ in prostate epithelium results in invasive and highly metastatic prostate carcinogenesis, and preferential malignant transformation of prostate stem cell compartment by combined deficiency by $\mathrm{p} 53$ and $\mathrm{Rb}$ indicates a critical role of these genes in the regulation of prostate stem cells during ontogenesis. Moreover, the loss of Pten/TP53 in prostate epithelium led to transformation of multipotential progenitors and EMT (41). These findings provide evidences that loss of WT-p53 may promote the metastasis of PCa by elevating migration, invasion, EMT and stemness of cancer cells.

Recently studies have found that miRNAs contribute to downregulation of mRNA and protein expression observed after p53 activation (24) and several miRNAs, which were the direct transcriptional targets of p53, played a critical role in mediating p53 regulation of EMT in cancer progression (8-11). The miR-34a, a tumor suppressor which directly targets Snail, was decreased due to the absence of WT-p53 function, thus Snail-dependent EMT was activated in colon, breast and lung carcinoma cells (22). The p53 also played a role in regulating EMT through transcriptional activation of miR-200c (8). Inhibition or overexpression of the miR-200 family affected p53-regulated EMT by altering ZEB1 and ZEB2 expression (10). Mutant p53 gain-of-function induces EMT through modulation of the miR-130b-ZEB1 axis in endometrial cancer (42). Previous studies have demonstrated that miR-145 is transcriptionally and post-transcriptionally regulated by WT-p53 (27-30), and miR-145 augments the effects of p53 by suppressing the inhibitors of p53 in cervical cancer cells (29). In this study, we found that WT-p53 enhanced miR-145 expression in PC-3 cells and anti-miR-145 reversed EMT features of PC-3 cells which were inhibited by ectopic expression of WT-p53. Importantly, miR-145 is upregulated by WT-p53, but not with mutant p53 in PC-3 cells (30), the anti-miR-145 also repressed the expression of WT-p53, and miR-145 itself also represses EMT in PC-3 cells (25). Collectively, these findings indicate that miR-145 is a mediator of WT-p53-regulated EMT.

Although miR-34a and miR-200c mediated p53-regulated EMT of cancer cells mainly through targeting EMT regulators Snail1, ZEB1 and ZEB2 $(8,10,42)$, our results suggested that miR-145 might mediate p53-regulated EMT of PC-3 cells by 
targeting several pathways. We found that WT-p53 repressed expression of the mesenchymal markers $\mathrm{N}$-cadherin, which was one of miR-145 targets (43), and ZEB2, which was speculated as one of miR-145 targets and a transcription factor promoting EMT, and the inhibitory effects of WT-p53 on expression of $\mathrm{N}$-cadherin and ZEB2 in PC-3 cells were reversed by antimiR-145. We also found that metastasis-promoting protein HEF1 was a direct target of miR-145 and it promoted EMT of PC-3 cells (the results not shown). Therefore, miR-145 may mediate WT-p53-regulated EMT of PCa cells by targeting $\mathrm{N}$-cadherin, ZEB2 and HEF1.

Besides regulating EMT, miRNAs which are the direct transcriptional targets of $\mathrm{p} 53$ played a role in mediating $\mathrm{p} 53$ regulation of cancer cell stemness in cancer progression. Liu et al (21) found that miR-34a inhibited prostate cancer stem cells and metastasis by directly repressing CD44. Furthermore, p53 regulated stem cell properties through modulating miR-200c by regulating KLF4 and BMI1 (8). Our previous results showed that miR-145 repressed stemness of PC-3 cells by suppressing CD44, Oct4, c-Myc and Klf4 (26). In present study, we showed that anti-miR-145 reversed colony formation and tumor sphere formation of PC-3 cells which were inhibited by ectopic expression of WT-p53. Moreover, WT-p53 also suppressed Oct4, c-Myc and Klf4 in PC-3 cells, which were directly targeted by miR-145 (27,31), CD44, which was speculated as one of miR-145 putative targets (miRWalk), and the inhibitory effect of WT-p53 on the above-mentioned 'stemness' factors is reversed by anti-miR-145. All these findings indicate that miR-145 also is a mediator of WT-p53 regulation of cancer cell stemness and suggest that miR-145 may target CD44, Oct4, c-Myc and Klf4 in PC-3 cells.

Recent evidence has demonstrated that the EMT can generate cancer cells with properties of stem cells $(44,45)$. This important finding implies a direct link between EMT and cancer stem cells. A previous study found that miR-200 played a critical role in linking EMT phenotype with stem cell signatures by regulating the expression of Lin28B and Notch1 in PCa cells (45). We found that p53/miR-145 pathway regulated both EMT and stemness of PC-3 cells. These finding suggested that the $\mathrm{p} 53 / \mathrm{miR}-145$ pathway might be the new link between EMT and cancer cell stemness in PC- 3 cells. Thus, the discovery of molecular targets which are regulated by $\mathrm{p} 53 / \mathrm{miR}-145$ axis and linked to EMT and CSC properties are required.

In conclusion, our findings demonstrate that WT-p53 suppresses migration, invasion, EMT and stemness of PC-3 PCa cells at least partially through modulating miR-145. These results suggest that loss of WT-p53 might promote bone metastasis of PCa at least partially through repressing miR-145 to elevate EMT and stemness of cancer cells. Therefore, the activation of the $\mathrm{p} 53 / \mathrm{miR}-145$ regulatory axis may function as a therapeutic alternative for bone metastasis of PCa.

\section{Acknowledgements}

We thank NSFC funding, China (no. 81272938); Science and Technology planning project of Guangdong Province, China (no. 2010B010800019); and Science and Technology Planning Project of Guangzhou, China (no. 11C22060772) for supporting this study.

\section{References}

1. Carlin BI and Andriole GL: The natural history, skeletal complications, and management of bone metastases in patients with prostate carcinoma. Cancer 88: 2989-2994, 2008.

2. Vousden KH and Prives C: Blinded by the light: the growing complexity of p53. Cell 137: 413-431, 2009.

3. Goh AM, Coffill CR and Lane DP: The role of mutant p53 in human cancer. J Pathol 223: 116-126, 2011.

4. Muller PA, Vousden KH and Norman JC: p53 and its mutants in tumor cell migration and invasion. J Cell Biol 192: 209-218, 2011.

5. Navone NM, Labate ME, Troncoso P, Pisters LL, Conti CJ, von Eschenbach AC and Logothetis CJ: p53 mutations in prostate cancer bone metastases suggest that selected p53 mutants in the primary site define foci with metastatic potential. J Urol 161: 304-308, 1999.

6. Wang SP, Wang WL, Chang YL, et al: p53 controls cancer cell invasion by inducing the MDM2-mediated degradation of Slug. Nat Cell Biol 11: 694-704, 2009.

7. Boominathan L: The guardians of the genome (p53, TA-p73, and TA-p63) are regulators of tumor suppressor miRNAs network. Cancer Metastasis Rev 29: 613-639, 2010.

8. Chang CJ, Chao $\mathrm{CH}$, Xia W, et al: p53 regulates epithelial-mesenchymal transition and stem cell properties through modulating miRNAs. Nat Cell Biol 13: 317-323, 2011.

9. Kim NH, Kim HS, Li XY, et al: A p53/miRNA-34 axis regulates Snaill-dependent cancer cell epithelial-mesenchymal transition. J Cell Biol 195: 417-433, 2011.

10. Kim T, Veronese A, Pichiorri F, et al: p53 regulates epithelial-mesenchymal transition through microRNAs targeting ZEB1 and ZEB2. J Exp Med 208: 875-883, 2011.

11. Schubert J and Brabletz T: p53 spreads out further: suppression of EMT and stemness by activating miR-200c expression. Cell Res 21: 705-707, 2011.

12. Hanahan D and Weinberg RA: Hallmarks of cancer: the next generation. Cell 144: 646-674, 2011.

13. Sethi S, Macoska J, Chen W and Sarkar FH: Molecular signature of epithelial-mesenchymal transition (EMT) in human prostate cancer bone metastasis. Am J Transl Res 3: 90-99, 2010.

14. Hugo H, Ackland ML, Blick T, Lawrence MG, Clements JA, Williams ED and Thompson EW: Epithelial-mesenchymal and mesenchymal-epithelial transitions in carcinoma progression. J Cell Physiol 213: 374-383, 2007.

15. Visvader JE and Lindeman GJ: Cancer stem cells in solid tumours: accumulating evidence and unresolved questions. Nat Rev Cancer 8: 755-768, 2008.

16. Chaffer CL and Weinberg RA: A perspective on cancer cell metastasis. Science 331: 1559-1564, 2011.

17. Monteiro $\mathbf{J}$ and Fodde R: Cancer stemness and metastasis: therapeutic consequences and perspectives. Eur J Cancer 46: 1198-1203, 2010.

18. Bracken CP, Gregory PA, Khew-Goodall Y and Goodall GJ: The role of microRNAs in metastasis and epithelial-mesenchymal transition. Cell Mol Life Sci 66: 1682-1699, 2009.

19. Gregory PA, Bert AG, Paterson EL, et al: The miR-200 family and miR-205 regulate epithelial to mesenchymal transition by targeting ZEB1 and SIP1. Nat Cell Biol 10: 593-601, 2008.

20. Korpal M, Lee ES, Hu G and Kang Y: The miR-200 family inhibits epithelial-mesenchymal transition and cancer cell migration by direct targeting of E-cadherin transcriptional repressors ZEB1 and ZEB2. J Biol Chem 283: 14910-14914, 2008.

21. Liu C, Kelnar K, Liu B, et al: The microRNA miR-34a inhibits prostate cancer stem cells and metastasis by directly repressing CD44. Nat Med 17: 211-215, 2011.

22. Siemens H, Jackstadt R, Hünten S, Kaller M, Menssen A, Götz U and Hermeking H: miR-34 and SNAIL form a doublenegative feedback loop to regulate epithelial-mesenchymal transitions. Cell Cycle 10: 4256-4271, 2011.

23. Hatfield S and Ruohola-Baker H: microRNA and stem cell function. Cell Tissue Res 331: 57-66, 2008.

24. Hermeking H: MicroRNAs in the p53 network: micromanagement of tumour suppression. Nat Rev Cancer 12: 613-626, 2012.

25. Peng X, Guo W, Liu T, et al: Identification of miRs-143 and -145 that is associated with bone metastasis of prostate cancer and involved in the regulation of EMT. PLoS One 6: e20341, 2011.

26. Huang S, Guo W, Tang Y, Ren D, Zou X and Peng X: miR-143 and miR-145 inhibit stem cell characteristics of PC-3 prostate cancer cells. Oncol Rep 28: 1831-1837, 2012. 
27. Sachdeva M, Zhu S, Wu F, et al: p53 represses c-Myc through induction of the tumor suppressor miR-145. Proc Natl Acad Sci USA 106: 3207-3212, 2009.

28. Suzuki HI, Yamagata K, Sugimoto K, Iwamoto T, Kato S and Miyazono K: Modulation of microRNA processing by $\mathrm{p} 53$ Nature 460: 529-533, 2009.

29. Shi M, Du L, Liu D, et al: Glucocorticoid regulation of a novel HPV-E6-p53-miR-145 pathway modulates invasion and therapy resistance of cervical cancer cells. J Pathol 228 148-157, 2011

30. Suh SO, Chen Y, Zaman MS, et al: MicroRNA-145 is regulated by DNA methylation and p53 gene mutation in prostate cancer. Carcinogenesis 32: 772-778, 2011.

31. Schmittgen TD and Livak KJ: Analysis of relative gene expression data using real-time quantitative PCR and the 2(-Delta Delta C (T)) method. Methods 25: 402-408, 2011.

32. Pfeiffer MJ and Schalken JA: Stem cell characteristics in prostate cancer cell lines. Eur Urol 57: 246-254, 2010.

33. Bisson I and Prowse DM: WNT signaling regulates self-renewal and differentiation of prostate cancer cells with stem cell characteristics. Cell Res 19: 683-697, 2009.

34. Xu N, Papagiannakopoulos T, Pan G, Thomson JA and Kosik KS: MicroRNA-145 regulates OCT-4, SOX2, and KLF4 and represses pluripotency in human embryonic stem cells. Cell 137: 647-658, 2009

35. Downing S, Bumak C, Nixdorf S, Ow K, Russell P and Jackson P: Elevated levels of prostate-specific antigen (PSA) in prostate cancer cells expressing mutant p53 is associated with tumor metastasis. Mol Carcinog 38: 130-140, 2003.

36. Stapleton AM, Timme TL, Gousse AE, et al: Primary human prostate cancer cells harboring p53 mutations are clonally expanded in metastases. Clin Cancer Res 3: 1389-1397, 1997.

37. Kogan-Sakin I, Tabach Y, Buganim Y, et al: Mutant p53 $(\mathrm{R} 175 \mathrm{H})$ upregulates Twist 1 expression and promotes epithelial-mesenchymal transition in immortalized prostate cells. Cell Death Differ 18: 271-281, 2011.
38. Vinall RL, Chen JQ, Hubbard NE, Sulaimon SS, Shen MM, Devere White RW and Borowsky AD: Initiation of prostate cancer in mice by Tp53R270H: evidence for an alternative molecular progression. Dis Model Mech 5: 914-920, 2012.

39. Zhou Z, Flesken-Nikitin A, Corney DC, Wang W, Goodrich DW, Roy-Burman P and Nikitin AY: Synergy of p53 and Rb deficiency in a conditional mouse model for metastatic prostate cancer. Cancer Res 66: 7889-7898, 2006.

40. Zhou Z, Flesken-Nikitin A and Nikitin AY: Prostate cancer associated with p53 and Rb deficiency arises from the stem/ progenitor cell-enriched proximal region of prostatic ducts. Cancer Res 67: 5683-5690, 2007.

41. Martin P, Liu YN, Pierce R, et al: Prostate epithelial Pten/TP53 loss leads to transformation of multipotential progenitors and epithelial to mesenchymal transition. Am J Pathol 179: 422-435, 2011.

42. Dong P, Karaayvaz M, Jia N, et al: Mutant p53 gain-of-function induces epithelial-mesenchymal transition through modulation of the miR-130b-ZEB1 axis. Oncogene: Jul 30, 2012 (Epub ahead of print). doi: 10.1038/onc.2012.334.

43. Gao P, Xing AY, Zhou GY, et al: The molecular mechanism of microRNA-145 to suppress invasion-metastasis cascade in gastric cancer. Oncogene 32: 491-501, 2013.

44. Mani SA, Guo W, Liao MJ, et al: The epithelial-mesenchymal transition generates cells with properties of stem cells. Cell 133: 704-715, 2008

45. Kong D, Banerjee S, Ahmad A, Li Y, Wang Z, Sethi S and Sarkar FH: Epithelial to mesenchymal transition is mechanistically linked with stem cell signatures in prostate cancer cells. PLoS One 5: e12445, 2010. 